\title{
Effects of Betaine Aldehyde Dehydrogenase-Transgenic Soybean on Phosphatase Activities and Rhizospheric Bacterial Community of the Saline-Alkali Soil
}

\author{
Ying Nie, ${ }^{1,2}$ Da-qing Wang, ${ }^{2}$ Guang Zhao, ${ }^{3}$ Song Yu, ${ }^{4}$ and Hong-yan Wang ${ }^{1}$ \\ ${ }^{1}$ College of Resources and Environment, Northeast Agricultural University, Harbin 150030, China \\ ${ }^{2}$ Heilongjiang State Farms Institute of Economy, Harbin 150090, China \\ ${ }^{3}$ School of Chemical \& Environmental Engineering, Liaoning University of Technology, Jinzhou, Liaoning 121001, China \\ ${ }^{4}$ College of Agriculture, Heilongjiang Bayi Agricultural University, Daqing, Heilongjiang 163319, China
}

Correspondence should be addressed to Hong-yan Wang; why220@126.com

Received 21 March 2016; Revised 11 July 2016; Accepted 18 July 2016

Academic Editor: Jiangke Yang

Copyright (C) 2016 Ying Nie et al. This is an open access article distributed under the Creative Commons Attribution License, which permits unrestricted use, distribution, and reproduction in any medium, provided the original work is properly cited.

\begin{abstract}
The development of transgenic soybean has produced numerous economic benefits; however the potential impact of root exudates upon soil ecological systems and rhizospheric soil microbial diversity has also received intensive attention. In the present study, the influence of saline-alkali tolerant transgenic soybean of betaine aldehyde dehydrogenase on bacterial community structure and soil phosphatase during growth stages was investigated. The results showed that, compared with nontransgenic soybean as a control, the rhizospheric soil $\mathrm{pH}$ of transgenic soybean significantly decreased at the seedling stage. Compared to HN35, organic P content was $13.5 \%$ and $25.4 \%$ greater at the pod-filling stage and maturity, respectively. The acid phosphatase activity of SRTS was significantly better than $\mathrm{HN} 35$ by $12.74 \%$ at seedling, $14.03 \%$ at flowering, and $59.29 \%$ at podding, while alkaline phosphatase achieved maximum activity in the flowering stage and was markedly lower than HN35 by $13.25 \%$ at pod-filling. The 454 pyrosequencing technique was employed to investigate bacterial diversity, with a total of 25,499 operational taxonomic units (OTUs) obtained from the 10 samples. Notably, the effect of SRTS on microbial richness and diversity of rhizospheric soil was marked at the stage of podding and podfilling. Proteobacteria, Acidobacteria, and Actinobacteria were the dominant phyla among all samples. Compared with HN35, the relative abundance of Proteobacteria was lower by $2.01 \%, 2.06 \%$, and $5.28 \%$ at the stage of seedling, at pod-bearing, and at maturity. In genus level, the relative abundance of Gp6, Sphingomonas sp., and GP4 was significantly inhibited by SRTS at the stage of podbearing and pod-filling.
\end{abstract}

\section{Introduction}

Genetically modified (GM) plants have been widely commercialized throughout the world, and more than 160 million hectares have been released for cultivation of GM plants [1]. In the meantime, the potential risk of horizontal gene transfer to the environment has received intensive attention from many scientists, who have studied, for example, the impact of GM on soil processes and microbial community diversity of rhizospheric soil [2]. Transgenic soybean is one of the most important GM plants, and its planting area reached about 70 million hectares in 2009 [3]. The betaine aldehyde dehydrogenase- (BADH-) transgenic soybean is genetically modified by betaine aldehyde dehydrogenase, which possesses a tolerance to saline-alkali soils. The BADH gene has a resistance to saline-alkali stress and drought stress and has transformed many crops including rice, maize, and potato, among others [4].

According to FAO (Food and Agriculture Organization) statistics, there are more than 900 million hectares of salinealkaline soils, mainly in arid and semiarid regions of the world $[5,6]$. Songnen Plain is one of the three primary contiguous saline-alkaline soil regions of the world, with an area of 3.73 million hectares in northeastern China. Due to a high 
$\mathrm{pH}$, high sodium absorption ratio, poor soil structure, and the low nutrient level of saline-alkaline soils, crop productivity and therefore economic development are intensively restricted. Therefore, soybean which is moderately tolerant of salinity is a feasible crop for Songnen Plain, as it is rich both in nutritional value and in great potential economic benefits. Although BADH-transgenic soybean has a tolerance to saline-alkaline stress, there is potential for root exudates to alter the soil chemistry process and soil microbial functions. In many countries, the pressure to improve crop production to satisfy human demands leads to approval of policies of planting saline-alkali tolerant plants, without then addressing the influence of these crops on the soil ecological process $[7,8]$.

Most studies concerning soil biological safety of transgenic plants are focused on the diversity of soil microbial community, such as soil enzyme activities, root exudates, and chemical processes of rhizosphere soil [9-11]. The soil ecosystem is important for nutrition cycle, surrounding minerals, and energy conversion and exchange. However, the potential risks of GM plants to ecological, environmental, and human health have become a public concern in recent years [12]. The rhizosphere is considered the most influential element of soil formation, which is intensively influenced by root secretions and associated bacterial diversity and functions [13]. Soil microbial enzyme activities, especially for functional groups of bacteria involved in $\mathrm{C}, \mathrm{N}$, and $\mathrm{P}$ cycling, are stimulated in the rhizosphere [14]. Biological and biochemical properties of soil have often been proposed as early sensitive indicators of soil ecological stress and other environmental changes. Generally, dehydrogenase and phosphatase enzyme activities in soil are closely related to the build-up of organic matter and provide sensitive information on the microbial activity of soil, which is thought to reflect the total range of oxidative activity of soil microflora, and consequently may be a good indicator of microbiological activity [15]. The types of root exudates and residual plant materials have an important impact on microbials residing in the rhizosphere. Any change in the quality and quantity of root exudates could potentially modify the composition and activity of soil microbials and may cause changes in both deleterious and beneficial microorganisms. This supports the idea that genetic modification of plants or microorganisms may cause shifts in microbial communities [16]. The BADHtransgenic soybean will not only greatly increase soybean production, but also promote the biological improvement for saline-alkaline soil in Songnen Plain. Nevertheless, few environment assessments have been reported for transgenic soybean expressing the BADH gene under field conditions.

Next-generation sequencing of 454 pyrosequencing technology is a powerful approach for studying bacterial diversity in various environment samples, such as soil and wastewater $[17,18]$. In this study, the growth stage-related dynamics of rhizospheric soil $\mathrm{pH}$ and phosphatase activities were evaluated. Moreover, 454 pyrosequencing technology was employed to investigate potential effects on the bacterial composition and diversity in the rhizosphere of transgenic soybean lines expressing the spinach betaine aldehyde dehydrogenase gene, compared to the nontransgenic parental cultivar at different plant development stages under field conditions of saline-alkaline soil in Songnen Plain. The results from this study will contribute to the assessment of environmental risks of transgenic crops to the soil ecosystem.

\section{Materials and Methods}

2.1. Study Site and Plants. The transgenic plants and control plants were grown on Anda farm at $46^{\circ} 01^{\prime}$ north and $124^{\circ} 55^{\prime}$ longitude located in Songnen Plain, Northeast China. The experimental soil is operated at a long-term (25 years) unfertilized and uncropped treatment of saline-alkali plot, and the plot was subdivided into 12 subplots $(10 \mathrm{~m} \times 6.5 \mathrm{~m}$ each). The trial soil belongs to the soda-saline soil, and initial characteristics of the surface were as follows: subclass: $38.9 \%$ sand, $39.7 \%$ silt, and $21.3 \%$ clay; $\mathrm{pH}: 8.34 \pm 0.06$; total organic carbon: $3.92 \pm 0.04 \mathrm{~g} \mathrm{~kg}^{-1}$; total nitrogen $(\mathrm{N}): 1.16 \pm$ $0.03 \mathrm{~g} \mathrm{~kg}^{-1}$ and $45.35 \pm 0.53 \mathrm{mg} / \mathrm{kg}$ of alkali-hydrolysable $\mathrm{N}$; total phosphorus (P): $0.62 \pm 0.01 \mathrm{~g} \mathrm{~kg}^{-1}$ and $25.79 \pm 0.33 \mathrm{mg} / \mathrm{kg}$ of available P; $148.28 \pm 2.06 \mathrm{mg} / \mathrm{kg}$ of available potassium.

The transgenic soybeans used in the experiments were $B A D H$-transgenic soybean (SRTS) and non- $B A D H$ transgenic soybean (HN35), which were provided by Heilongjiang Academy of Agricultural Sciences, Harbin, China.

2.2. Experimental Design and Soil Sampling. The rhizosphere soil samples were collected at five different stages of plant growth: 25, 50, 75, 100, and 125 days after sowing (DAS). At every sampling stage, five randomly chosen plants per subplot were removed and the adhering soil was placed in sterile plastic bags ( $\approx 2.5 \mathrm{~kg}$ of moist soil) from six replicate plots of soybean in the same day; after removal of roots the soil samples were homogenized and were pooled together in order to obtain a representative sample for library construction. The samples were transported to the laboratory in dry ice chests within 2 hours. Then, one portion of the composite soil was used for the enzyme activity measurements, while other smaller amounts of the soil ( $10 \mathrm{~g})$ were separated, stored in centrifuge tubes, and kept at $-20^{\circ} \mathrm{C}$ in the dark until DNA extraction and further processing.

2.3. Determination of Soil $p H$ and Phosphatase Enzyme Activity. The rhizospheric soil $\mathrm{pH}$ was determined using $\mathrm{pH}$ meter (320-S, Mettler-Toledo Instruments, Co., Ltd., Shanghai, China) with $1: 1$ ratio water/soil at room temperature $\left(25^{\circ} \mathrm{C}\right)$. Acid phosphatase activity and alkaline phosphatases activity derived from rhizospheric soil were measured according to the method by Tabatabai [19]. All of the values were calculated in triplicate.

\subsection{Microbial Community Analysis}

2.4.1. DNA Extraction from Rhizosphere Soil Samples. Soil genomic DNA was extracted directly from the rhizosphere soil samples using the soil DNA kit (Omega Biotek, Inc., Norcross, GA, USA) according to the manufacturer's instructions. The amount, quality, and purity of the extracted soil 
DNA were checked by electrophoresis in 1\% agarose gels in $1 \mathrm{x}$ TBE buffer, run for $1 \mathrm{~h}$ at $90 \mathrm{~V}$, and stained with ethidium bromide, upon exposure to UV.

2.4.2. PCR Amplification and Pyrosequencing. PCR was performed using the primers $341 \mathrm{f}\left(5^{\prime}\right.$-GCCTCCCTCGCGCCAT-CAGNNNNNNNCCTACGGGAGGCAGCAG$\left.3^{\prime}\right)$ and 534r(5'-GCCTTGCCAGCCCGCTCA GNNNNNNNATTACCGCGGCTGCTGG-3'), in which the italicized sequence is that of a 454 Life Sciences ${ }^{\circledR}$ primer and the underlined sequence is a barcode sequence tag. Amplification was performed in a $50 \mu \mathrm{L}$ reaction volume, which contained $100 \mathrm{ng}$ genomic DNA, 1x reaction buffer, $3 \mathrm{mM} \mathrm{MgCl}_{2}, 20 \mathrm{pmol}$ each primer, $0.4 \mathrm{mM}$ (each) dNTP, and $1.25 \mathrm{U}$ of Taq DNA polymerase. The amplification conditions consisted of an initial denaturation step of $94^{\circ} \mathrm{C}$ for $3 \mathrm{~min}$, followed by 30 cycles of $94^{\circ} \mathrm{C}$ for $30 \mathrm{~s}, 56^{\circ} \mathrm{C}$ for $30 \mathrm{~s}$, and $72^{\circ} \mathrm{C}$ for $45 \mathrm{~s}$, and a final elongation step at $72^{\circ} \mathrm{C}$ for $3 \mathrm{~min}$. All of the PCR reactions were conducted using a GeneAmp PCR System 9700 (Applied Biosystems, Foster City, CA, USA).

2.4.3. Statistical and Bioinformatics Analysis. The microbial sequences were sorted into each sample batch using the barcode tag in the Pipeline Initial Process at the RDP, and the RDP classifier (http://rdp.cme.msu.edu/classifier/classifier .jsp) was applied to classify the sequence data from genus to phylum at hierarchical levels [20]. Distance matrices and statistical parameters, including rarefaction curves, ShannonWeaver indices, Simpson's diversity (1-D), coverage estimators (SACE), and Chaol (SChaol), were calculated using Mothur software [21].

\section{Results and Discussion}

3.1. Dynamic Change of Rhizosphere Soil $p H$. The rhizospheric soil $\mathrm{pH}$ variation of transgenic soybean (SRTS) and nontransgenic soybean (HN35) exhibited similar trends during the whole growth period. Compared with HN35, the $\mathrm{pH}$ of SRTS was lower at 25, 50, 75, and 100 days after sowing time (Figure 1). The pH of SRTS (8.19) was significantly lower than for HN35 at seedling stage. However, with the growth of the soybean, the SRTS pH eventually exceeded that of HN35 at maturity stage, which might be due to the amount of root exudates produced. Therefore, more $\mathrm{H}^{+}$secretion could be released by SRTS rhizosphere and cause $\mathrm{pH}$ to decrease under the stress of a saline-alkali and low phosphorus environment. The population of bacteria and the phosphorus availability of SRTS rhizospheric soil likely increased due to the decrease of $\mathrm{pH}$ at the seedling stage.

3.2. Effects of Transgenic Soybean on Organic P and Phosphatase Activities in Rhizospheric Soil. The organic P content in rhizospheric soil planted with SRTS and HN35 increased initially and then reached maximum content at pod-filling stage (Figure 2). The treatments of SRTS organic P were higher than for HN35 during growth periods but lower at the seeding stage. Compared to $\mathrm{HN} 35$, organic $\mathrm{P}$ was $13.5 \%$

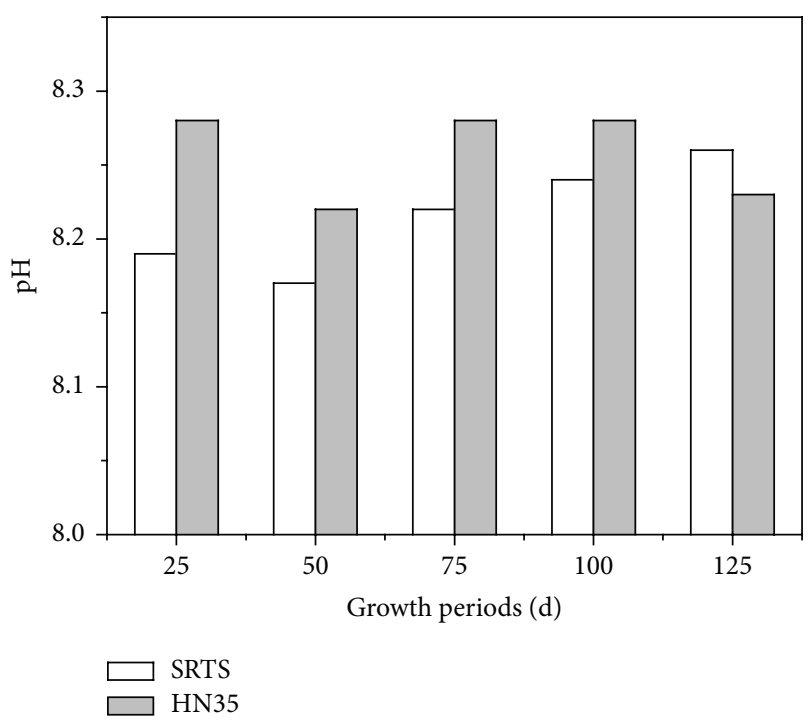

FIgURE 1: The $\mathrm{pH}$ of the rhizospheric soil of transgenic soybean and nontransgenic soybean at different growth stages.

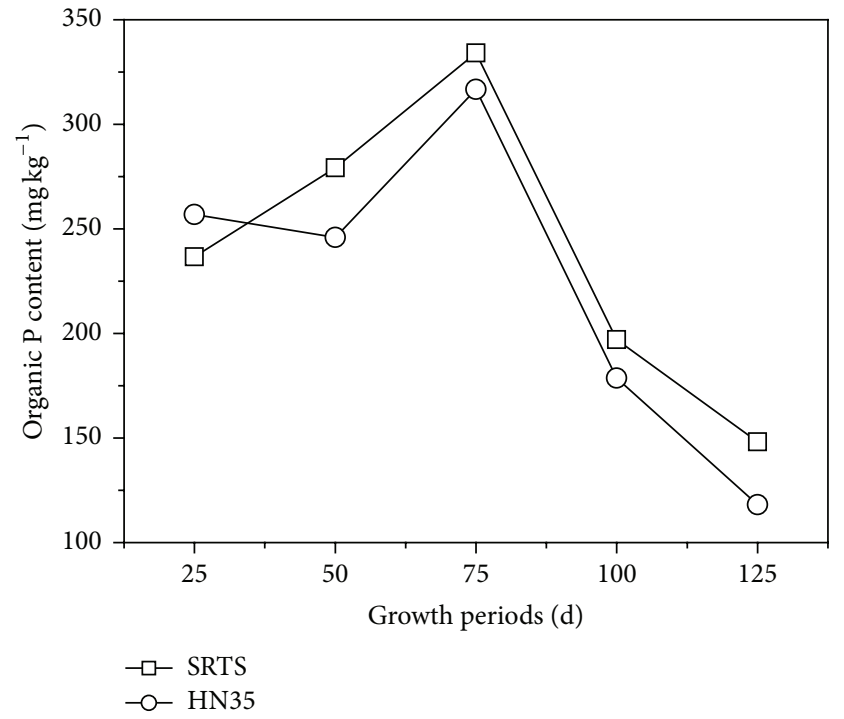

FIGURE 2: Organic P of the rhizospheric soil of transgenic soybean and nontransgenic soybean at different growth stages.

and $25.4 \%$ greater at pod-filling stage and maturity stage, respectively. Organic $\mathrm{P}$ is significant for immobilization and mineralization of soil P. Due to the low inorganic P content of saline-alkali soil, mineralization of organic $\mathrm{P}$ was supplied for the plant. The results showed that mineralization of organic P was significantly influenced by the growth stages of transgenic soybean.

The phosphatase activity controls the release of organic phosphorus and the biological validity of soil. Activity of acid phosphatase (a) and alkaline phosphatase (b) showed a similar trend in rhizospheric soil for SRTS and HN35 during different growth periods (Figure 3 ). The activity of acid phosphatases was highest at the seedling stage and gradually decreased with the growth of the soybean. The 


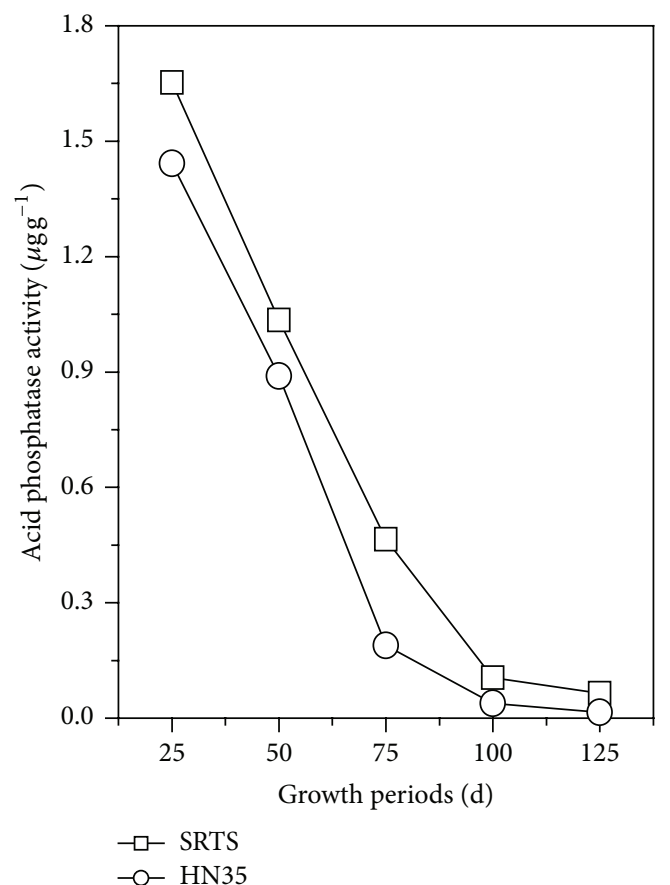

(a)

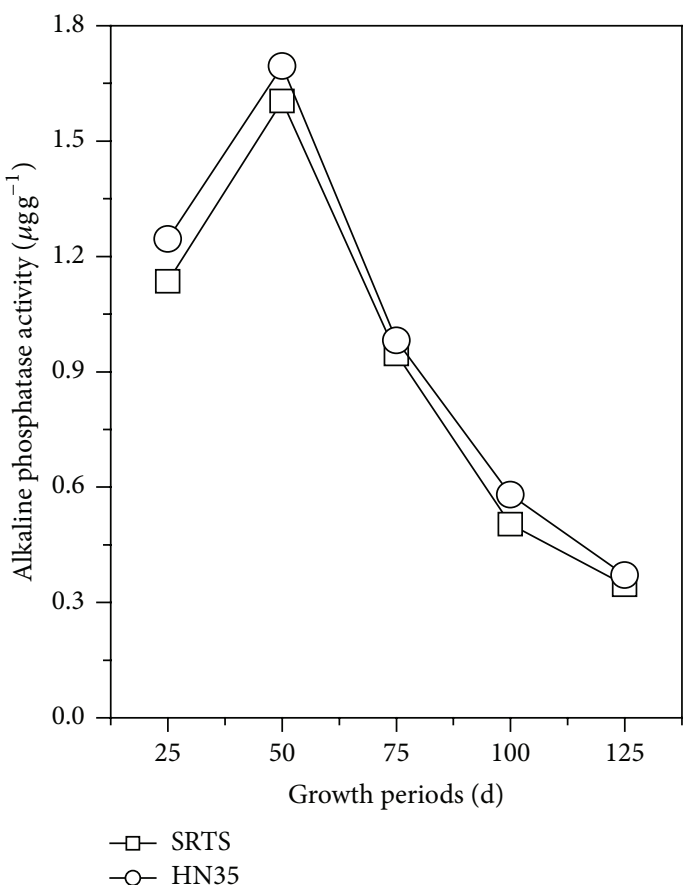

(b)

FIgURE 3: Activity of acid phosphatase (a) and alkaline phosphatase (b) in rhizospheric soil under transgenic soybeans and nontransgenic isolines during different growth stages.

acid phosphatase activity of SRTS was significantly higher than HN35 at the seedling, flowering, and podding stages in rhizosphere soil, at $12.74 \%, 14.03 \%$, and $59.29 \%$ higher, respectively. Therefore, the effect of SRTS on the activity of rhizosphere soil acid phosphatase was significantly influenced by changes in soybean growth stage. The activity of alkaline phosphatase achieved maximum effect in the flowering stage and decreased in the following growth stage. Compared with HN35, the effect of SRTS on rhizosphere soil alkaline phosphatase was markedly low and decreased by $13.25 \%$ in pod-filling stage.

\subsection{Effects of BADH Soybean on Bacterial Communities Diversity in the Rhizospheric Soil}

3.3.1. Sequences Analysis of Pyrosequencing. A total of 56,119 valid reads and 25,499 operational taxonomic units (OTUs) were obtained from the 10 samples of rhizospheric soil by 454 pyrosequencing analysis at $3 \%$ sequence similarity distance (Table 1). Rarefaction curves at $97 \%$ cut-off showed similar trends for all samples, yet none had reached saturation plateau (Figure 4). Despite examining nearly 5600 sequences of each sample, the ACE and Chaol indicated high diversity in the bacterial community of soybean rhizospheric soil. Therefore, deeper sequencing may detect new bacterial phenotypes in soybean rhizospheric soil samples. The average sample achieved at 3\% distance was estimated at $71.14 \%$ (Table 1 ). Notably, the effect of SRTS on richness and diversity of rhizospheric soil was marked in the podding and pod-filling stages. These changes in microbial diversity and enzyme

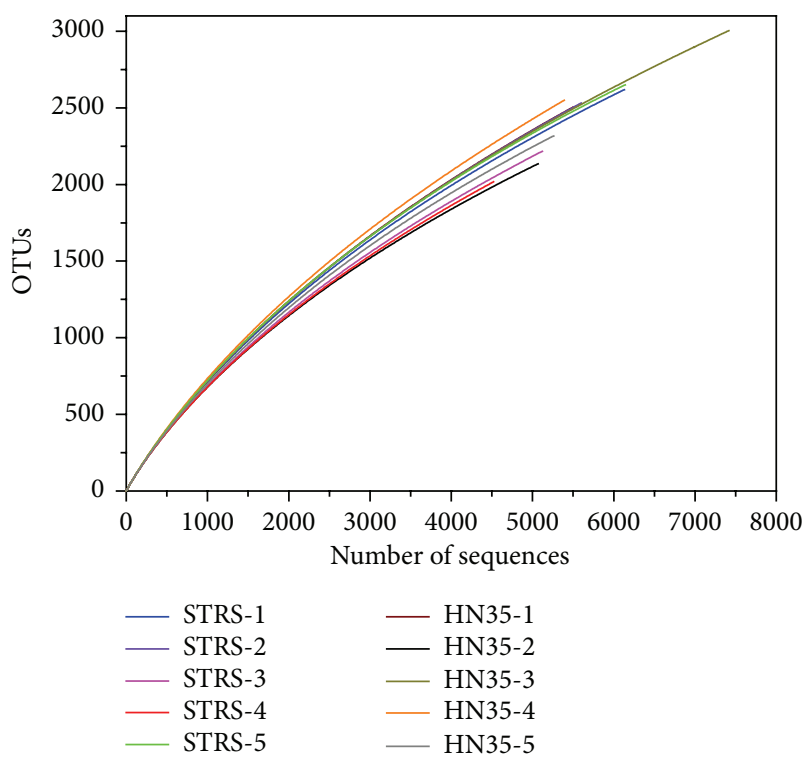

FIgURE 4: Rarefaction curves of sequences of $16 \mathrm{~S}$ rRNA. The rarefaction curves from rhizospheric soil samples of transgenic soybeans and nontransgenic soybeans were calculated by $97 \%$ sequence similarity during different growth periods. Note: SRTS and BADH (1-5) mean $25 \mathrm{~d}, 50 \mathrm{~d}, 75 \mathrm{~d}, 100 \mathrm{~d}$, and $125 \mathrm{~d}$ of different stages of soybean growth periods.

activity between SRTS and HN35 during the growth stage could probably cause shifts in microbial structures and biological process. 
TABLE 1: Comparison of the estimated operational taxonomic unit (OTU) richness and diversity indices of the 16S rRNA gene for clustering at $97 \%$ identity as obtained from the pyrosequencing analysis.

\begin{tabular}{|c|c|c|c|c|c|c|}
\hline \multirow{2}{*}{ Sample } & \multirow{2}{*}{${ }^{\mathrm{a}} \mathrm{NS}$} & \multirow{2}{*}{ boTUs } & \multicolumn{2}{|c|}{ Estimated OTU richness } & \multirow{2}{*}{ Shannon } & \multirow{2}{*}{$\begin{array}{c}\text { Coverage } \\
(\%)\end{array}$} \\
\hline & & & ACE & Chaol & & \\
\hline SRTS-1 & 6130 & 2722 & 7848 & 5455 & 7.2869 & 72.89 \\
\hline HN35-1 & 5500 & 2615 & 8863 & 5866 & 7.3031 & 69.20 \\
\hline SRTS-2 & 5596 & 2621 & 8510 & 5901 & 7.2914 & 69.94 \\
\hline HN35-2 & 5063 & 2233 & 7561 & 4840 & 7.1371 & 72.11 \\
\hline SRTS-3 & 5120 & 2284 & 7264 & 5074 & 7.1747 & 71.70 \\
\hline HN35-3 & 7418 & 3098 & 9602 & 6582 & 7.3845 & 74.31 \\
\hline SRTS-4 & 4518 & 2054 & 6376 & 4237 & 7.0772 & 71.51 \\
\hline HN35-4 & 5387 & 2624 & 9813 & 6324 & 7.3845 & 67.77 \\
\hline SRTS-5 & 6133 & 2768 & 8092 & 5880 & 7.3616 & 71.92 \\
\hline HN35-5 & 5254 & 2480 & 7742 & 5131 & 7.2727 & 70.14 \\
\hline
\end{tabular}

${ }^{\mathrm{a}}$ Number of sequences for sample.

${ }^{\mathrm{b}}$ Calculated with DOTUR at the $3 \%$ distance level.

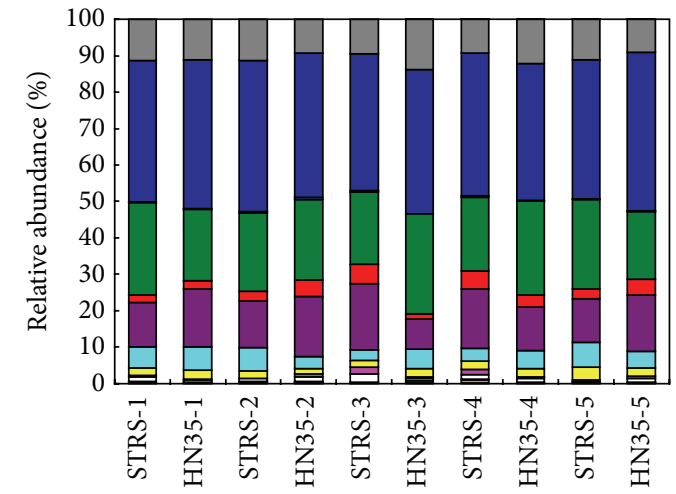

Phyla A

- Unclassified_Bacteria

- Proteobacteria

- Chloroflexi

- Acidobacteria

口 Firmicutes

- Actinobacteria

$\square$ Bacteroidetes

口 Gemmatimonadetes

๑ Candidatus Saccharibacteria $\square$ Armatimonadetes

(a)

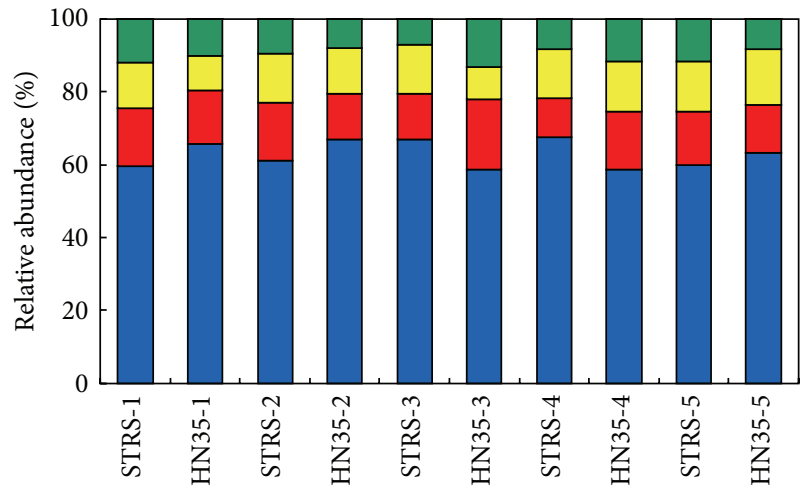

Phyla B

$\square$ Deltaproteobacteria $\square$ Gammaproteobacteria

$\square$ Betaproteobacteria $\square$ Alphaproteobacteria

Figure 5: Taxonomic composition of different phyla for the sequences retrieved from rhizospheric soil samples of transgenic soybeans and nontransgenic soybeans during the whole growth stage based on the classification of partial 16S rRNA sequences of bacteria using RDP classifier. Phyla A: Bacteria phylum distribution; Phyla B: Proteobacteria classes distribution.

3.3.2. Overall Phylogenetic Analysis of Bacterial Communities. Many previous studies found that different varieties of plants could affect the microbial diversity of rhizospheric soil, yet little credible research has been conducted into whether transgenic plants can affect enzyme activities and the diversity of indigenous microbial populations $[8,22,23]$. In this study, the bacterial community analysis of rhizospheric soil for SRTS and HN35 at different growth stages was assessed using pyrosequencing technology, grouping the sequences at phylum. Of the classifiable sequences, 18 phyla were identified, and the dominant phyla were Proteobacteria,
Acidobacteria, and Actinobacteria which were present in all samples (Figure 5(a)). Proteobacteria were the most dominant bacteria, which accounted for more than $38 \%$ of total sequences in all treatments. Compared with HN35, the relative abundance of Proteobacteria was $2.01 \%, 2.06 \%$, and $5.28 \%$ lower in seedling, pod-bearing, and maturity stages, respectively. The abundance of phylum Acidobacteria was also clearly changed in transgenic soybeans of SRTS compared with nontransgenic soybeans of HN35, greater by $5.73 \%$ in seedling stage and $6.02 \%$ in the maturing period, yet the relative abundance of Acidobacteria was lower by $7.98 \%$ 


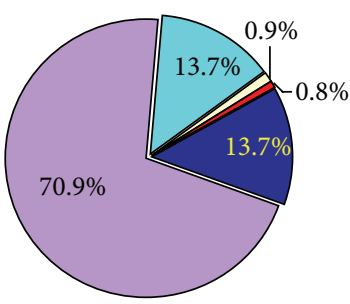

STRS-1

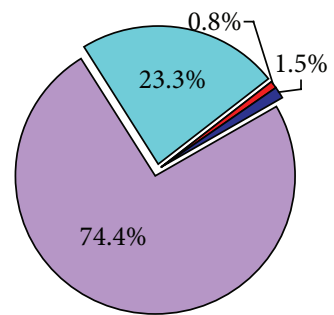

STRS-3

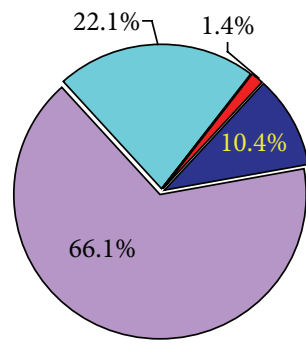

STRS-5

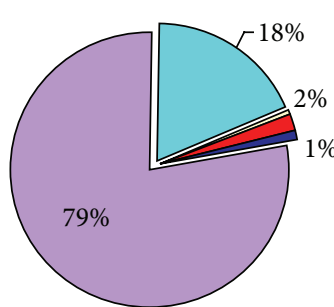

HN35-1

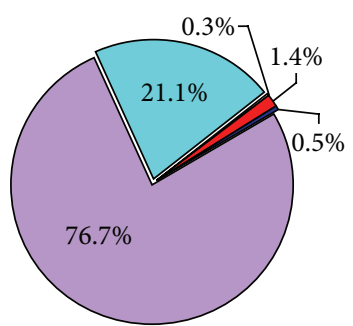

HN35-3

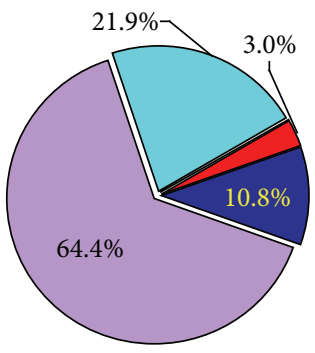

HN35-5

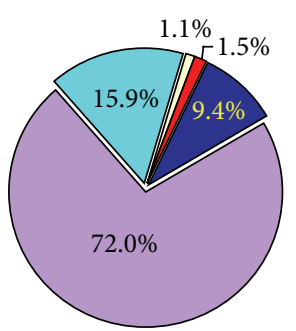

STRS-2

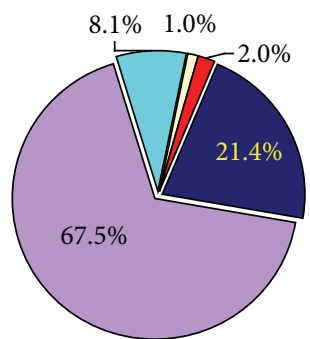

STRS-4
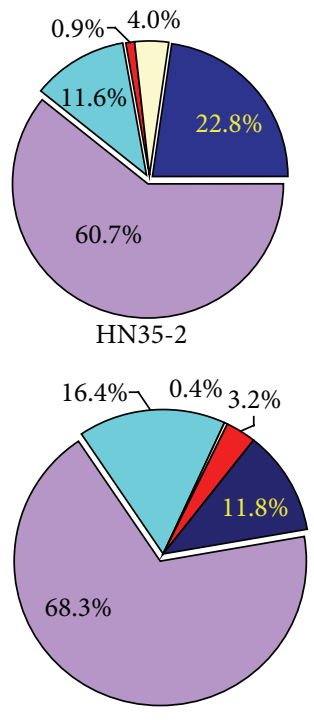

Sphingobium

Unclassified_Sphingomonadaceae

Sphingomonas

Sphingopyxis

Sandaracinobacter

FIGURE 6: The relative abundance of Sphingomonadaceae family level distribution in each sample based on the classification of partial 16S rRNA sequences.

and $5.41 \%$ in pod-bearing and pod-filling stages, respectively. The obvious change of Actinobacteria occurred in the podbearing stage, where the relative abundance accounted for $18.14 \%$ in SRTS yet decreased to $8.36 \%$ in HN35. Furthermore, some less dominant bacteria were observed during different growth stages, such as Bacteroidetes, Firmicutes, Gemmatimonadetes, Cyanobacteria, Candidatus Saccharibacteria, and Nitrospirae.

The most dominant class distribution of Proteobacteria observed was Alphaproteobacteria, which accounted for more than $58 \%$ of total sequences of all treatments (Figure 5(b)). The relative abundance of Alphaproteobacteria of SRTS was $6.15 \%, 6.04 \%$, and $3.23 \%$ lower than in HN35 at seedling, flowering, and maturity stages, respectively; however, its abundance was $8.23 \%$ and $8.89 \%$ greater at podbearing and pod-filling stages. Clear changes in abundance of Betaproteobacteria were evident in pod-bearing and podfilling stages compared with $\mathrm{HN} 35$, when relative abundance decreased by $6.75 \%$ and $5.28 \%$, respectively. Notably, the class Deltaproteobacteria was 6.32\% lower in SRTS than in $\mathrm{HN} 35$ at these growth stages and did not change significantly in other growth stages. In genus-level distribution of Sphingomonadaceae, Sphingomonas sp. dominated in all treatments, and Sphingopyxis sp. was the second predominant bacteria (Figure 6). It was clear that the relative abundance of STRS was $8.1 \%$ less than for HN35 at the seedling stage and $9.5 \%$ greater in the flowering stage.

In genus level, there were 32 genera found with a relative abundance exceeding $0.2 \%$ of total bacteria (Table 2). The dominant bacteria for STRS and HN35 were Gp6 (12.48\% and $8.89 \%$, resp.), Sphingomonas sp. (9.14\%, 7.35\%), Gp4 (5.37\%, 2.93\%), Gemmatimonas sp. $(2.14 \%, 2.31 \%), \mathrm{Gp} 7$ $(1.68 \%, 1.25 \%)$, Ohtaekwangia sp. (1.52\%, 1.80\%), and Gp16 $(1.24 \%, 1.04 \%)$ at seedling stage (relative abundance $\geq 1.0 \%)$. Notably, Gp6, Gp4, Gp7, and Gp16 belonged to Acidobacteria, dominating $20.77 \%$ and $14.11 \%$ of total sequences for STRS and HN35 at seeding stage. This was probably the reason that $\mathrm{pH}$ of SRTS rhizospheric soil was significantly lower than that for HN35 in seedling stage.

The relative abundance of genus Gp6, Sphingomonas sp., and GP4 was significantly inhibited by STRS compared with HN35. Gp6 was lower by $12.38 \%$ and $9.02 \%$ at pod-bearing 
TABLe 2: Phylogenetic classification of genus for the sequences retrieved from rhizospheric soil samples of transgenic soybeans and nontransgenic soybeans during different growth stages (relative abundance $>0.18 \%$ ).

\begin{tabular}{|c|c|c|c|c|c|c|c|c|c|c|}
\hline \multirow{2}{*}{ Classification } & \multicolumn{10}{|c|}{ Phylogenetic classification of clusters of sample (\%) } \\
\hline & SRTS-1 & HN35-1 & SRTS-2 & HN35-2 & SRTS-3 & HN35-3 & SRTS-4 & HN35-4 & SRTS-5 & HN35-5 \\
\hline Gp6 & 12.48 & 8.89 & 9.72 & 4.50 & 2.99 & 15.37 & 2.08 & 11.10 & 11.19 & 5.06 \\
\hline Sphingomonas & 9.14 & 7.35 & 7.90 & 3.40 & 2.54 & 8.29 & 3.32 & 7.43 & 6.13 & 5.10 \\
\hline Gp4 & 5.37 & 2.93 & 3.40 & 1.90 & 1.02 & 5.24 & 0.84 & 5.10 & 6.33 & 1.98 \\
\hline Gemmatimonas & 2.14 & 2.31 & 2.11 & 1.66 & 1.80 & 2.32 & 2.26 & 2.26 & 3.49 & 2.36 \\
\hline Gp7 & 1.68 & 1.25 & 0.91 & 0.75 & 0.37 & 1.78 & 0.38 & 2.00 & 1.24 & 0.69 \\
\hline Ohtaekwangia & 1.52 & 1.80 & 0.89 & 0.12 & 0.25 & 1.02 & 0.24 & 0.84 & 1.42 & 0.55 \\
\hline Gp16 & 1.24 & 1.04 & 1.18 & 0.73 & 0.53 & 1.46 & 0.62 & 1.21 & 0.73 & 0.74 \\
\hline Kofleria & 1.00 & 0.69 & 0.61 & 0.47 & 0.45 & 0.92 & 0.62 & 0.84 & 0.75 & 0.67 \\
\hline Azospirillum & 0.95 & 1.87 & 0.95 & 0.59 & 0.61 & 1.02 & 0.31 & 0.54 & 1.76 & 0.32 \\
\hline Rubrobacter & 0.80 & 1.84 & 0.66 & 0.34 & 0.29 & 0.50 & 0.29 & 0.37 & 0.82 & 0.19 \\
\hline Thermoleophilum & 0.70 & 0.45 & 0.36 & 0.41 & 0.53 & 0.31 & 0.27 & 0.33 & 0.31 & 0.38 \\
\hline Phenylobacterium & 0.57 & 0.58 & 0.54 & 0.83 & 0.66 & 0.38 & 0.64 & 0.48 & 0.24 & 0.36 \\
\hline Ramlibacter & 0.49 & 0.58 & 0.41 & 0.14 & 0.06 & 0.74 & 0.22 & 0.45 & 0.29 & 0.23 \\
\hline Chitinophaga & 0.47 & 0.53 & 0.68 & 0.32 & 0.23 & 0.50 & 0.40 & 0.26 & 0.38 & 0.27 \\
\hline Lysobacter & 0.47 & 0.45 & 0.55 & 0.22 & 0.16 & 0.36 & 0.11 & 0.37 & 0.62 & 1.50 \\
\hline Flavitalea & 0.46 & 0.33 & 0.50 & 0.16 & 0.10 & 0.38 & 0.33 & 0.67 & 0.68 & 0.21 \\
\hline Geminicoccus & 0.46 & 0.67 & 0.38 & 0.34 & 0.31 & 0.32 & 0.11 & 0.22 & 0.36 & 0.17 \\
\hline Devosia & 0.44 & 0.71 & 0.52 & 0.71 & 0.78 & 0.26 & 1.37 & 0.52 & 0.29 & 0.80 \\
\hline Microvirga & 0.38 & 1.25 & 0.45 & 0.14 & 0.25 & 0.36 & 0.13 & 0.37 & 0.55 & 0.29 \\
\hline Adhaeribacter & 0.36 & 0.58 & 0.36 & 0.02 & 0.14 & 0.39 & 0.04 & 0.30 & 0.21 & 0.15 \\
\hline Bauldia & 0.33 & 0.45 & 0.38 & 0.08 & 0.23 & 0.24 & 0.20 & 0.07 & 0.29 & 0.08 \\
\hline Streptophyta & 0.29 & 0.15 & 0.21 & 0.61 & 0.98 & 0.05 & 0.53 & 0.17 & 0.13 & 0.36 \\
\hline Dyella & 0.28 & 0.18 & 0.20 & 0.97 & 0.66 & 0.03 & 0.66 & 0.19 & 0.08 & 0.40 \\
\hline Solirubrobacter & 0.28 & 0.22 & 0.27 & 0.34 & 0.53 & 0.11 & 0.77 & 0.28 & 0.08 & 0.40 \\
\hline Roseomonas & 0.26 & 0.35 & 0.36 & 0.61 & 1.25 & 0.20 & 0.89 & 0.28 & 0.15 & 0.74 \\
\hline Ralstonia & 0.24 & 0.58 & 0.36 & 1.17 & 0.96 & 0.13 & 0.55 & 0.17 & 0.08 & 0.95 \\
\hline Dongia & 0.24 & 0.20 & 0.20 & 0.10 & 0.06 & 0.44 & 0.20 & 0.17 & 0.47 & 0.29 \\
\hline Steroidobacter & 0.24 & 0.15 & 0.38 & 0.16 & 0.23 & 0.38 & 0.11 & 0.26 & 0.29 & 0.36 \\
\hline Aquicella & 0.23 & 0.11 & 0.16 & 0.28 & 0.27 & 0.18 & 0.20 & 0.22 & 0.15 & 0.17 \\
\hline Mesorhizobium & 0.21 & 0.18 & 0.16 & 0.91 & 0.61 & 0.16 & 0.55 & 0.32 & 0.08 & 0.65 \\
\hline Nocardioides & 0.20 & 0.31 & 0.14 & 0.26 & 0.27 & 0.16 & 0.66 & 0.24 & 0.21 & 0.27 \\
\hline Lactobacillus & 0.18 & 0.18 & 0.23 & 0.63 & 0.49 & 0.04 & 0.64 & 0.43 & 0.10 & 0.27 \\
\hline Psychrobacter & 0.18 & 0.13 & 0.29 & 0.40 & 0.39 & 0.04 & 0.35 & 0.07 & 0.08 & 0.49 \\
\hline Hyphomicrobium & 0.18 & 0.27 & 0.34 & 0.75 & 0.35 & 0.11 & 0.64 & 0.24 & 0.28 & 0.38 \\
\hline
\end{tabular}

stage and pod-filling stages; however, it was $6.03 \%$ greater in the maturing period. Genus Sphingomonas sp. accounted for $2.54 \%$ of bacteria in the pod-bearing stage, when minimum relative abundance was $5.75 \%$ lower than for HN35. The trend was opposite to that of the flowering stage, compared with HN35, when Sphingomonas sp. increased by $4.5 \%$. The relative abundance of genus Gemmatimonas sp. was not significant between STRS and HN35 during the growth stage.

The principal component analysis based on weighted UniFrac distance was used to reveal the relationships among the 10 samples at the $97 \%$ similarity of sequences (Figure 7). The principal component analysis showed that STRS-5 and NH35-5, STRS-1, NH35-4, STRS-2, NH35-1, and NH35-3 were grouped to the right of the principal component, which represented $14.8 \%$ of the total variation. STRS-3, STRS-4, and NH35-2 were grouped into a cluster near the principal component and accounted for $14.4 \%$.

\section{Conclusions}

The potential risk of BADH-transgenic soybean to soil ecosystems has aroused great attention in recent years. In this study, the $\mathrm{pH}$, organic $\mathrm{P}$ content, and phosphatase activity of rhizospheric soil were used to assess soil process changes between SRTS and HN35 over a growth period of time. Furthermore, the 454 pyrosequencing technique was employed to expose the soil microbial community. The results suggested that root exudates of BADH-transgenic soybean had a significant impact on phosphatase activity and bacterial diversity of rhizospheric soil at different growth 


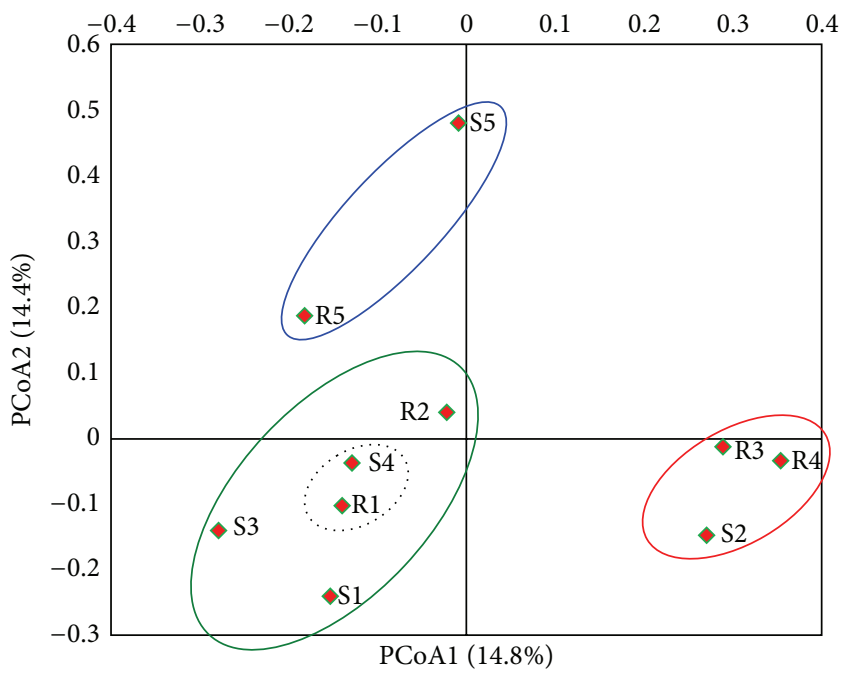

Figure 7: PCoA analysis of the bacteria of each sample at the 97\% similarity of sequences based on UniFrac method. Principal component 1 and principal component 2 explained $14.8 \%$ and $14.4 \%$ of the total variations. Note: R1-R5 and S1-S5 mean $25 \mathrm{~d}, 50 \mathrm{~d}, 75 \mathrm{~d}$, $100 \mathrm{~d}$, and $125 \mathrm{~d}$ of STRS and NH35 of soybean at different growth stages.

stages. Compared to HN35, acid phosphatase activity of SRTS was significantly greater $(59.29 \%)$ at the stage of podding, which accorded with organic $\mathrm{P}$ content variation, while alkaline phosphatase was markedly lower for SRTS by $13.25 \%$ at the stage of pod-filling. Bacterial richness and diversity were markedly influenced by SRTS at the stage of podding and pod-filling. Genus of Gp6, Sphingomonas sp., and GP4 were significantly inhibited by STRS at the stage of podbearing and pod-filling. Therefore, further research should focus on evaluating biological safety based on soil processes and change of soil microbial function.

\section{Competing Interests}

The authors declare that they have no competing interests.

\section{Acknowledgments}

The authors are grateful for support by the project of Chinese National Major Projects for Science and Technology Development (2009ZX08011-025B-02) and Scientific Research Project Funds for Graduate Innovation of Heilongjiang Province, China (YJSCX2012-045HLJ).

\section{References}

[1] C. James, Global Status of Commercialized Biotech/GM Crops, 43, International Service for the Acquisition of AgriBiotech Applications, 2011.

[2] Y.-E. Lee, S.-H. Yang, T.-W. Bae, H.-G. Kang, P.-O. Lim, and H.Y. Lee, "Effects of field-grown genetically modified Zoysia grass on bacterial community structure," Journal of Microbiology and Biotechnology, vol. 21, no. 4, pp. 333-340, 2011.
[3] C. James, "Global status of commercialized biotech/GM crops," ISAAA Brief 41, ISAAA, Metro Manila, Philippines, 2009.

[4] W. Fan, M. Zhang, H. Zhang, and P. Zhang, "Improved tolerance to various abiotic stresses in transgenic sweet potato (Ipomoea batatas) expressing spinach betaine aldehyde dehydrogenase," PLoS ONE, vol. 7, no. 5, Article ID e37344, 2012.

[5] W. Shi, T. Takano, and S. Liu, "Isolation and characterization of novel bacterial taxa from extreme alkali-saline soil," World Journal of Microbiology and Biotechnology, vol. 28, no. 5, pp. 2147-2157, 2012.

[6] H. Ma, H. Yang, X. Lü et al., "Does high $\mathrm{pH}$ give a reliable assessment of the effect of alkaline soil on seed germination? A case study with Leymus chinensis (Poaceae)," Plant and Soil, vol. 394, no. 1-2, pp. 35-43, 2015.

[7] K. E. Dunfield and J. J. Germida, "Impact of genetically modified crops on soil- and plant-associated microbial communities," Journal of Environmental Quality, vol. 33, no. 3, pp. 806-815, 2004.

[8] Z. H. Chen, L. J. Chen, and Z. J. Wu, "Relationships among persistence of Bacillus thuringiensis and Cowpea trypsin inhibitor proteins, microbial properties and enzymatic activities in rhizosphere soil after repeated cultivation with transgenic cotton," Applied Soil Ecology, vol. 53, no. 1, pp. 23-30, 2012.

[9] A. L. Cerdeira, D. L. P. Gazziero, S. O. Duke, M. B. Matallo, and C. A. Spadotto, "Review of potential environmental impacts of transgenic glyphosate-resistant soybean in Brazil," Journal of Environmental Science and Health Part B: Pesticides, Food Contaminants, and Agricultural Wastes, vol. 42, no. 5, pp. 539549, 2007.

[10] H. Fang, B. Dong, H. Yan, F. Tang, B. Wang, and Y. Yu, "Effect of vegetation of transgenic Bt rice lines and their straw amendment on soil enzymes, respiration, functional diversity and community structure of soil microorganisms under field conditions," Journal of Environmental Sciences, vol. 24, no. 7, pp. 1259-1270, 2012.

[11] U. Mina and A. Chaudhary, "Impact of transgenic cotton varieties on activity of enzymes in their rhizosphere," Indian Journal of Biochemistry \& Biophysics, vol. 49, no. 3, pp. 195-201, 2012.

[12] J. D. Wolt and R. K. D. Peterson, "Prospective formulation of environmental risk assessments: probabilistic screening for CrylA(b) maize risk to aquatic insects," Ecotoxicology and Environmental Safety, vol. 73, no. 6, pp. 1182-1188, 2010.

[13] P. Čapek, K. Diáková, J.-E. Dickopp et al., “The effect of warming on the vulnerability of subducted organic carbon in arctic soils," Soil Biology and Biochemistry, vol. 90, pp. 19-29, 2015.

[14] S. R. Cotta, A. C. Franco Dias, I. E. Marriel, F. D. Andreote, L. Seldin, and J. D. van Elsas, "Different effects of transgenic maize and nontransgenic maize on nitrogen-transforming archaea and bacteria in tropical soils," Applied and Environmental Microbiology, vol. 80, no. 20, pp. 6437-6445, 2014.

[15] V. Acosta-Martínez, S. E. Dowd, Y. Sun, D. Wester, and V. Allen, "Pyrosequencing analysis for characterization of soil bacterial populations as affected by an integrated livestockcotton production system," Applied Soil Ecology, vol. 45, no. 1, pp. 13-25, 2010.

[16] V. Chaudhry, A. Rehman, A. Mishra, P. S. Chauhan, and C. S. Nautiyal, "Changes in bacterial community structure of agricultural land due to long-term organic and chemical amendments," Microbial Ecology, vol. 64, no. 2, pp. 450-460, 2012. 
[17] D. L. Kirchman, M. T. Cottrell, and C. Lovejoy, “The structure of bacterial communities in the western Arctic Ocean as revealed by pyrosequencing of $16 \mathrm{~S}$ rRNA genes," Environmental Microbiology, vol. 12, no. 5, pp. 1132-1143, 2010.

[18] P. Trivedi, Z. He, J. D. Van Nostrand, G. Albrigo, J. Zhou, and $\mathrm{N}$. Wang, "Huanglongbing alters the structure and functional diversity of microbial communities associated with citrus rhizosphere," ISME Journal, vol. 6, no. 2, pp. 363-383, 2012.

[19] M. A. Tabatabai, "Soil enzymes," in Methods of Soil Analysis. Part 2, J. M. Bigham, Ed., Book Series No. 5, pp. 775-834, SSSA, Madison, Wis, USA, 1994.

[20] Q. Wang, G. M. Garrity, J. M. Tiedje, and J. R. Cole, "Naïve Bayesian classifier for rapid assignment of rRNA sequences into the new bacterial taxonomy," Applied and Environmental Microbiology, vol. 73, no. 16, pp. 5261-5267, 2007.

[21] P. D. Schloss, S. L. Westcott, T. Ryabin et al., "Introducing mothur: open-source, platform-independent, communitysupported software for describing and comparing microbial communities," Applied and Environmental Microbiology, vol. 75, no. 23, pp. 7537-7541, 2009.

[22] M. Castaldini, A. Turrini, C. Sbrana et al., "Impact of Bt corn on rhizospheric and soil eubacterial communities and on beneficial mycorrhizal symbiosis in experimental microcosms," Applied and Environmental Microbiology, vol. 71, no. 11, pp. 6719-6729, 2005.

[23] M. Wei, F. Tan, H. Zhu et al., "Impact of Bt-transgenic rice (SHK601) on soil ecosystems in the rhizosphere during crop development," Plant, Soil and Environment, vol. 58, no. 5, pp. 217-223, 2012. 

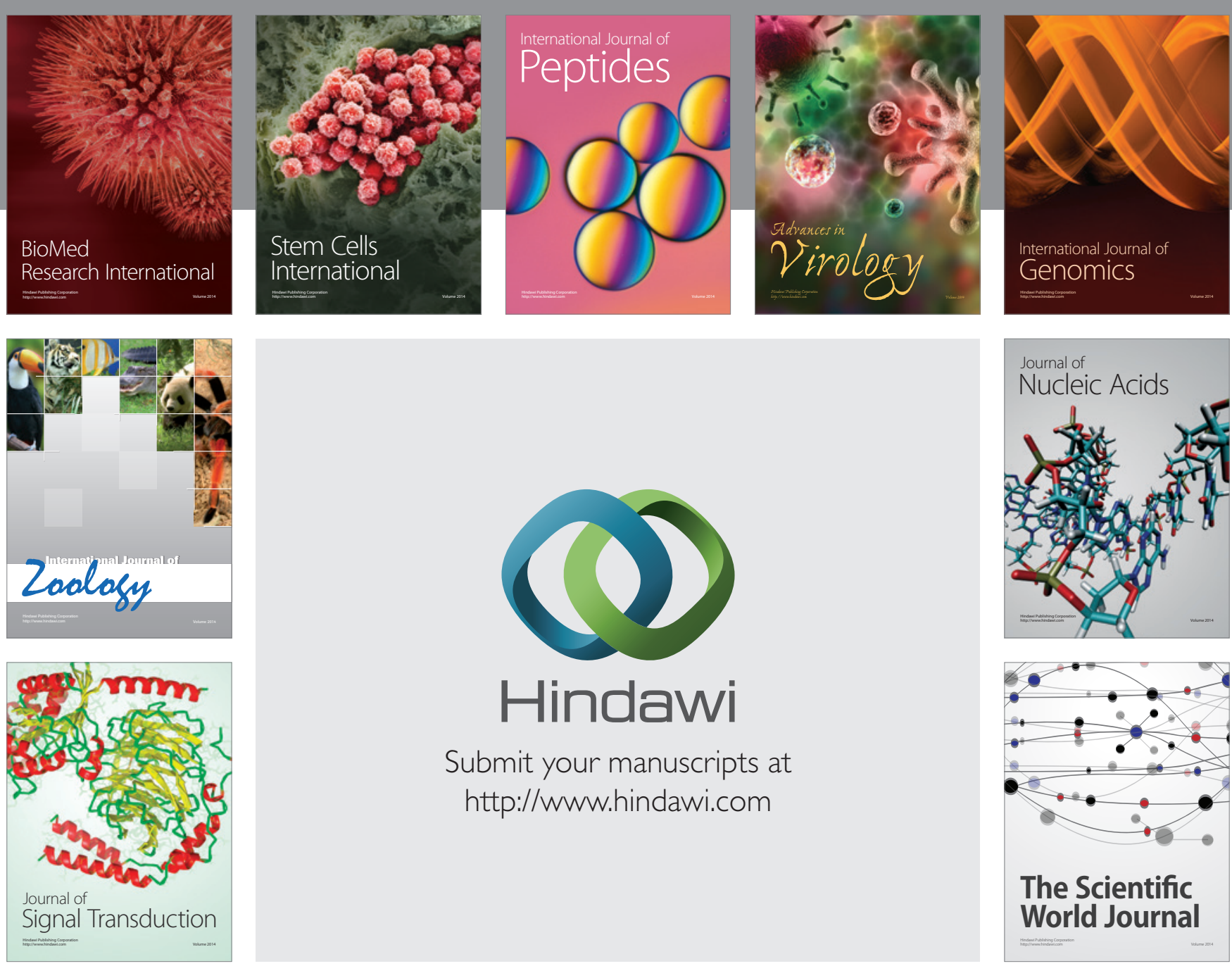

Submit your manuscripts at

http://www.hindawi.com
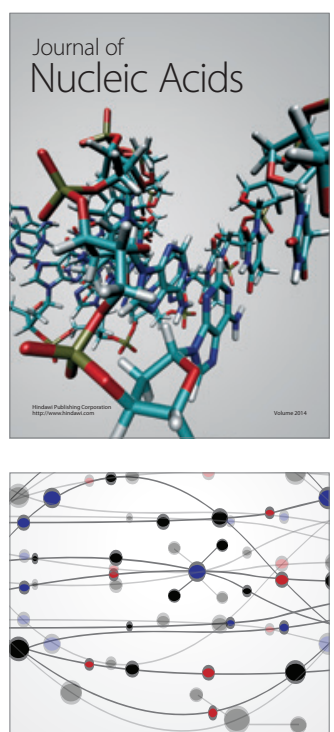

The Scientific World Journal
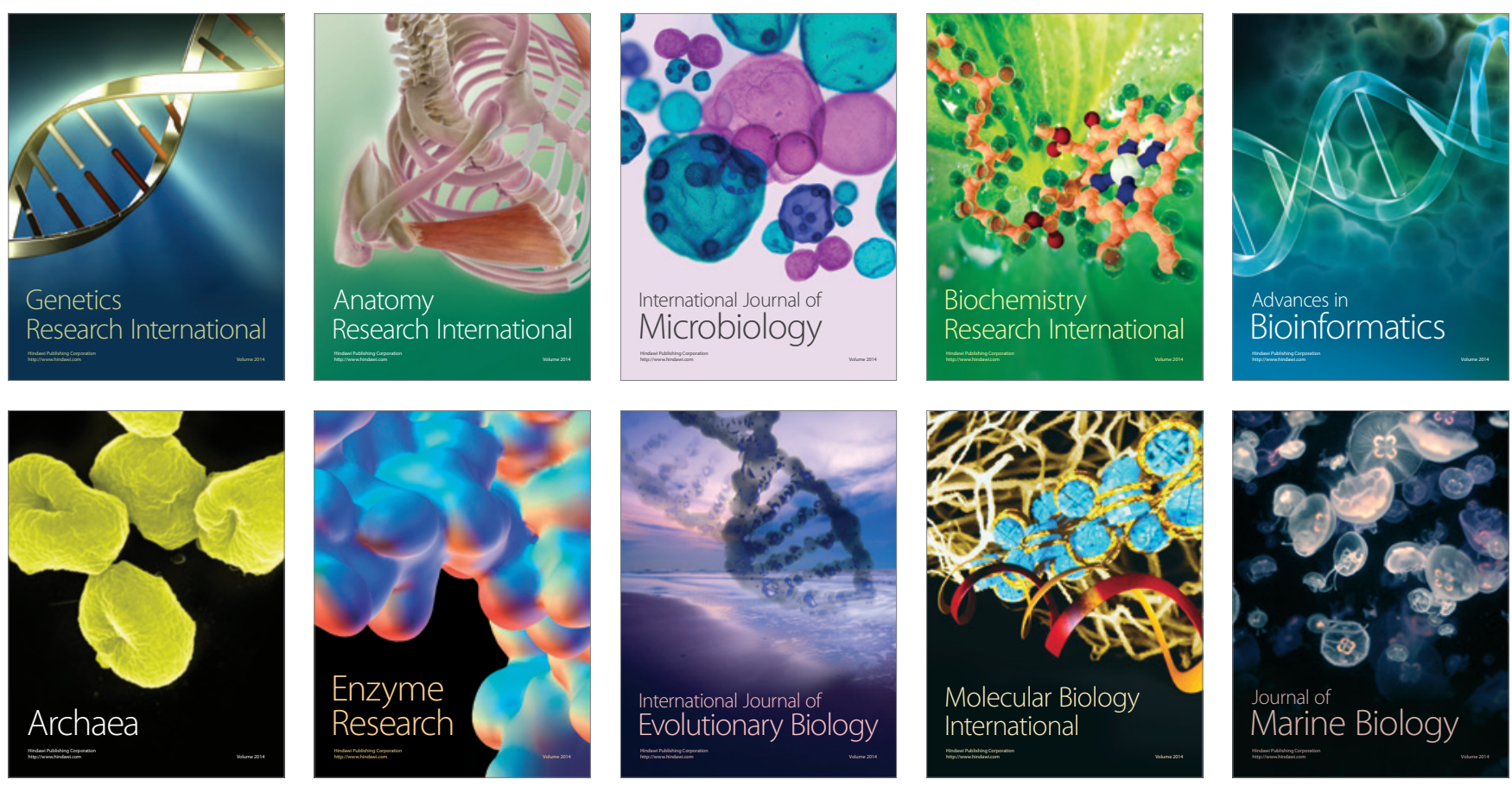